\title{
Ambion
}

THE RNA COMPANY

\section{MELT TM Total Nucleic Acid Isolation System: a new technology for hands-free tissue disruption, RNA preservation and total nucleic acid purification}

\author{
Gary J Latham \& Heidi J Peltier
}

Ambion's multi-enzymatic liquefaction of tissue (MELT ${ }^{T M}$ ) Total Nucleic Acid I solation System allows the hands-free disruption of several solid tissues in parallel without tissue grinding or the need for a polytron. Up to $10 \mathrm{mg}$ of fresh or frozen tissue can be lysed at room temperature in 5-15 min using a combination of powerful catabolic enzymes and a potent small-molecule RNase inhibitor. The procedure recovers highquality total RNA with two- to threefold better yield and $40 \%$ less hands-on time than present methods. Moreover, MELT reagents irreversibly inactivate RNases, allowing the storage of liquefied tissue for more than a week at room temperature without an appreciable loss of RNA integrity. RT-PCR and microarray expression profiling studies of MELT RNA reveal an excellent correlation with RNA extracted using popular methods, including those based on single-reagent phenol solutions and glass-filter purification. A simple alteration in the protocol also allows the recovery of genomic DNA.

Tissue is a complex repository of molecular clues that can guide the understanding, diagnosis and treatment of disease. In both research and clinical settings, diseased and normal tissues harbor genomic and proteomic profiles that 'fingerprint' their biological status ${ }^{1,2}$. Expression profiling tools, such as microarrays and real-time quantitative RT-PCR, can unveil highly specific patterns of gene expression that promise to link specific molecular events with the disease phenotype ${ }^{3}$.

Present approaches to gene expression analyses often hinge on the isolation of intact, high-quality RNA, which can serve as a 'snapshot' of the expression profile. The first step of most RNA extractions is the mechanical disruption of the sample tissue with a dounce, a polytron, a mill or a similar device. These methods are cumbersome, provide low throughput, require washing of the disruption apparatus between samples and are potentially inefficient. Such methods can also present a biological hazard by exposing the operator to aerosols from diseased samples.

\section{Hands-free, closed-tube tissue disruption}

The limitations of physical tissue disruption encouraged the development of a new technology to allow the extraction of RNA and DNA from solid tissues without the need for hands-on tissue grinding. Ambion's MELT Total Nucleic Acid Isolation System (patent pending) provides a unique set of reagents that include a potent

Ambion, Inc. 2130 Woodward, Austin, Texas 78744-1832, USA. Correspondence should be addressed to G.J.L. (glatham@ambion.com).

PUBLISHED ONLINE 23 AUGUST 2005; DOI:10.1038/ NMETH789 small-molecule RNase inhibitor and a cocktail of catabolic enzymes that can liquefy up to $10 \mathrm{mg}$ of tissue at room temperature in 5-15 min. The RNase inhibitor protects RNA from degradation during the early stages of tissue digestion, providing sufficient time for

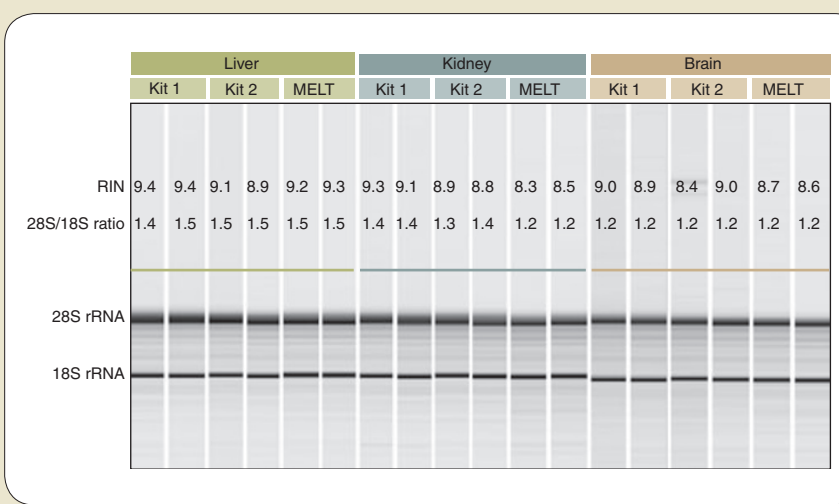

Figure 1 | High-quality RNA obtained using the MELT Total Nucleic Acid Isolation System. Total RNA was isolated from fresh mouse liver, kidney and brain tissue ( $\sim 5 \mathrm{mg}$ ) using MELT, or one of two competitor kits, Kit 1 (a single-reagent phenolic solution-based method) or Kit 2 (a glass fiber column-based method). RNA was isolated according to the supplier's protocol for each method except for an additional glass filter purification step for Kit 1. Samples were resolved by microcapillary gel electrophoresis, and the gels were scanned using an Agilent 2100 bioanalyzer. RIN and $28 \mathrm{~S} / 18 \mathrm{~S}$ ratio (indicated in each lane) were determined using the 2100 expert software (Agilent). 
APPLICATION NOTES

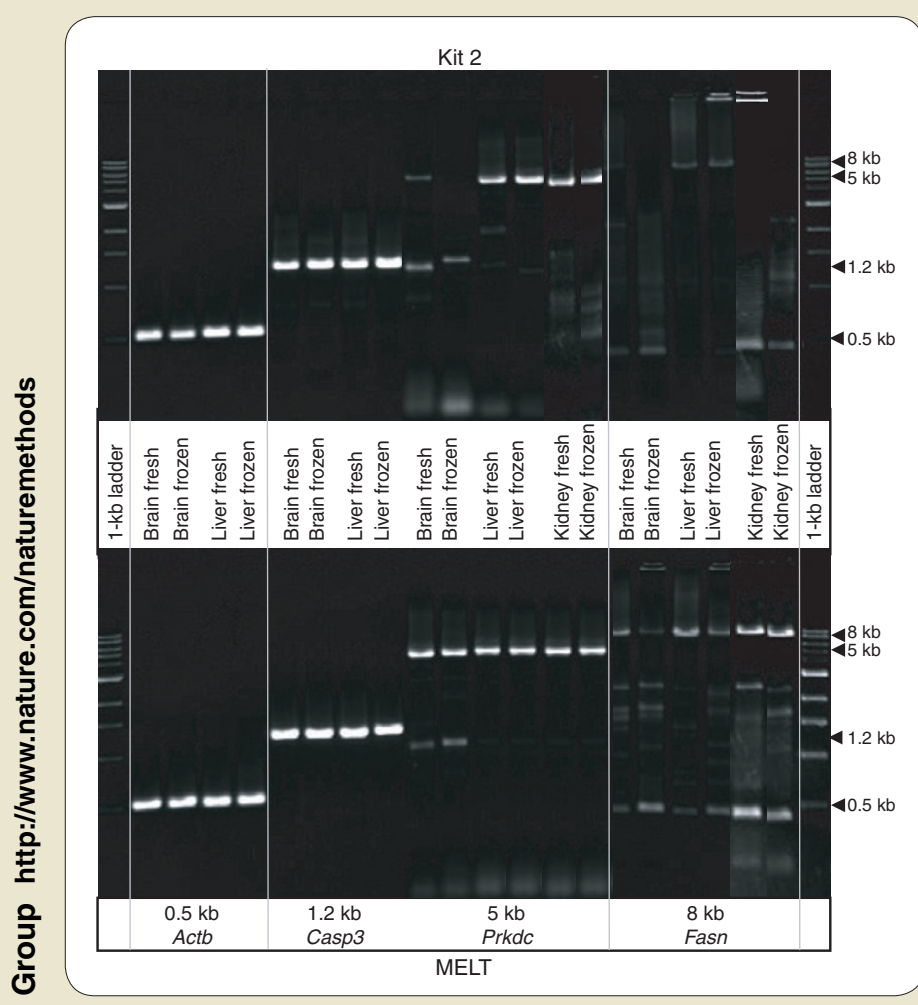

Figure 2 | RNA isolated from MELT lysates allows the efficient synthesis of amplicons as long as $8 \mathrm{~kb}$. Total RNA was isolated from $\sim 5 \mathrm{mg}$ of freshly collected or frozen mouse brain, liver and kidney tissues using two different methods: a guanidine-based glass filter method (Kit 2) or the MELT Total Nucleic Acid Isolation System. The reverse transcription (RT) of $1 \mu \mathrm{g}$ of total RNA was accomplished using ArrayScript ${ }^{T M}$ RT (Ambion) with an oligo(dT) primer in a 1 -h reaction at $42{ }^{\circ} \mathrm{C}$. This step was followed by a 40 -cycle PCR using a hotstart Taq polymerase. Products were resolved on a $1 \%$ agarose gel, and stained with SYBR Gold. All RT minus controls were negative for the specific products. Actb, $\beta$ actin; Casp3, Caspase-3; Prkdc, DNA-dependent protein kinase C; Fasn, fatty acid synthase.

the MELT enzymes to destroy the cellular architecture and unwanted macromolecules, including RNases. Both freshly collected and flash-frozen tissue can be used, and more than 20 mouse and human tissues - including tumor tissues — have been successfully liquefied using MELT reagents. Moreover, the MELT procedure can be used for fatty, fibrous and more routine soft tissues.

\section{Magnetic bead- based nucleic acid purification}

After digestion, total RNA is purified using a streamlined protocol based on Ambion's MagMAX ${ }^{\mathrm{TM}}$ magnetic bead procedure. This batch solution purification strategy eliminates the variability in sample processing introduced by filter clogging. RNA-binding magnetic beads are added to the MELT tissue lysate, and the particles are captured either in tubes using a stand magnet or in a 96-well plate with a matching magnetic plate. Cell debris and other contaminants are then washed away. The beads are next treated with Ambion's TURBO DNase ${ }^{\mathrm{TM}}$ to degrade genomic DNA. The fragmented DNA is removed in a subsequent wash step, and the purified RNA is eluted in as little as $20 \mu \mathrm{l}$ of elution solution. Genomic DNA can be purified using a slightly modified protocol that uses an in-line RNase digestion to remove RNA. The complete procedure, from tissue to a

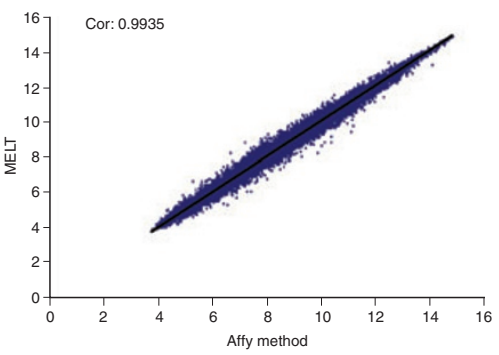

b

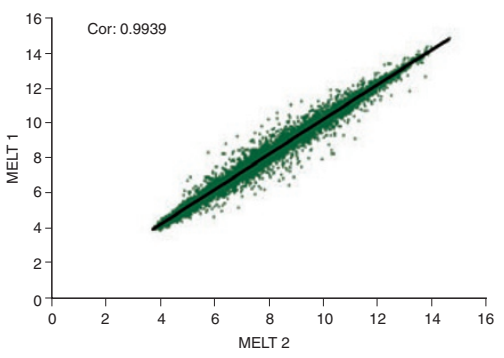

C

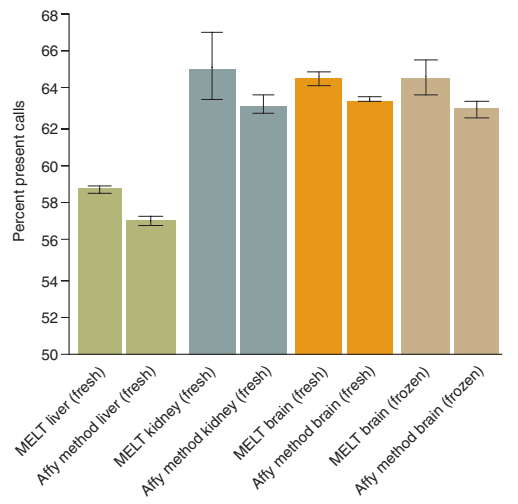

Figure 3 | Microarray analysis of RNA isolated from the MELT Total Nucleic Acid I solation System. (a-c) Total RNA was isolated from fresh mouse liver, kidney, and brain tissue ( $\sim 5 \mathrm{mg}$ ) using MELT or the method recommended in the Affymetrix GeneChip Expression Analysis Technical Manual (Comp. A). Total RNA ( $1 \mu \mathrm{g}$ ) was amplified using Ambion's MessageAmp II antisense RNA (aRNA) Amplification Kit. Fragmented aRNA was then hybridized to Mouse Genome 430A 2.0 arrays (representing $>14,500$ genes) and scanned with a GeneChip Scanner 3000. Data were captured and analyzed with the GeneChip Operating Software (Affymetrix). Results are shown after normalization of array signal intensities using robust multichip analysis. Biological replicates are represented between the two methods for fresh mouse liver and kidney, as well as for fresh and frozen mouse brain tissue. Pearson's correlation between results from fresh mouse liver RNA obtained using the MELT and Affymetrix recommended methods (a). Pearson's correlation within the MELT method with fresh mouse brain (b). Percent present calls obtained using either the MELT or the Affymetrix recommended isolation methods from the indicated fresh and frozen mouse tissues (c). Error bars represent s.d. of the present call values. Cor, Pearson's correlation coefficient.

purified DNA-free RNA (or RNA-free DNA), requires less than one hour to process 6-12 samples. Yields of RNA are typically two to three times higher than yields obtained using competing technologies.

\section{RNA integrity comparable with standard methods}

The goal of any RNA isolation procedure is to recover an RNA population that faithfully mirrors the biology of the sample at the time of 
procurement. Thus, it is critical that the sample preparation procedure itself does not compromise the integrity of the RNA. To ensure that RNA recovered after MELT digestion was intact, this RNA was compared with total RNA isolated using two popular methods, one using a single-reagent phenolic solution and another based on purification using glass-filter columns. Two different RNA quality metrics were used to evaluate the RNA: the 28S/18S rRNA ratio and the RNA integrity number (RIN), a new index that rates RNA integrity from 1 (worst) to 10 (best). RNA isolated from MELT lysates is characterized by $28 \mathrm{~S} / 18 \mathrm{~S}$ rRNA ratios of $\geq 1.2$ and RINs of 8.3-9.3, which compare favorably with assessments of RNA purified using the two comparison methods (Fig. 1).

\section{RT-PCR of MELT RNA}

Ambion scientists have extensively validated the MELT technology to ensure that the purified RNA is at least as functional as RNA isolated using popular methods. For example, RNA isolated from fresh and flash-frozen tissues from the MELT system is suitable for both endpoint RT-PCR and quantitative RT-PCR. Indeed, cDNA products as long as 8 kilobases ( $\mathrm{kb}$ ) can be amplified from total RNA isolated with the MELT System (Fig. 2). Only 1.3\% of all known mouse transcripts are longer than $8 \mathrm{~kb}$ (X. Wang, personal communication). Our data revealed that 0.5-, 1.2-, 5.0- and 8.0-kb amplicons can be successfully synthesized from RNA purified using the MELT system, whereas yields of the 5.0 - and $8.0-\mathrm{kb}$ products were much reduced using RNA that was isolated with glass filter columns (Kit 2). As a result, MELT RNA contains long, intact transcripts that can be readily amplified by RT-PCR.

The expression of six randomly selected genes (Actb, Myc, Jun, $\operatorname{Trp53}$, Casp3 and $C d k-9$ ) was measured in quantitative RT-PCR experiments that compared the products of the MELT procedure with two other popular RNA isolation approaches requiring sample homogenization and subsequent purification with a single-reagent phenol solution and/or glass filter columns. Quantitation of these transcripts across five mouse tissues (brain, liver, kidney, heart and intestine) did not reveal any significant difference among the methods, based on comparisons of cycle threshold values for each mRNA with the appropriate standards (data not shown). Moreover, the relative expression levels for each of the six transcripts were comparable for both fresh and flash-frozen tissues for all three RNA isolation procedures.

\section{Microarray analysis of MELT RNA}

Perhaps the most stringent test for RNA quality is to evaluate the RNA by microarray analysis. Commercially available microarrays contain thousands of gene elements that permit expression profiling from large portions of the host transcriptome. Whereas expression of a subset of mRNAs may be conserved across RNA samples of very different quality or content, global concordance among samples generally implies a high standard of RNA equivalence. Thus, RNA isolated after MELT was compared with RNA isolated after sample homogenization using the method recommended in the Affymetrix GeneChip Expression Analysis manual. This method is widely recognized as the most rigorous protocol for the recovery of highly intact RNA from solid tissues. Biological replicates of RNA were extracted from mouse liver, kidney and brain and amplified using an Eberwine amplification procedure ${ }^{4}$. Data from samples recovered using MELT revealed slightly higher percent present calls (Fig. 3) than the recommended method, and both cross-method and within-method replicates had signal correlation coefficients greater than 0.984 for all tissues. Additional analyses of other key array metrics confirmed that MELT RNA was highly concordant with RNA isolated using the present best methods.

\section{RNA preservation in tissue lysates}

A major threat to RNA integrity in tissues is RNase activity. Unlike chaotropes such as guanidine that transiently inhibit RNases, MELT reagents digest RNases to peptide fragments that cannot reactivate. As a result, MELT enzymes irreversibly destroy RNase activity and allow storage of intact RNA in tissue lysates for more than a week at ambient temperatures. RNA from tissue homogenates stored in guanidine-based lysis solutions, by comparison, is substantially degraded within $24-48 \mathrm{~h}$. The ability of the MELT kit reagents to achieve both tissue digestion and RNA preservation means that samples can be processed, conveniently stored and readily transported at ambient temperatures for downstream analyses.

\section{Conclusions}

The MELT Total Nucleic Acid Isolation System offers several key advantages over presently available RNA and DNA isolation kits. Most notable among these is the ability to lyse solid tissue samples without a homogenizer or any hands-on effort. Tissue is digested in minutes in a closed tube using a cocktail of catabolic enzymes and nuclease inhibitors. Because MELT reagents enzymatically cleave RNases to peptide fragments, the RNA in tissue lysates is stable for more than a week at ambient temperature. High yields of intact RNA and DNA are recovered after MELT tissue liquefaction using a magnetic bead-based protocol that expedites the processing of dozens of samples at a time. RNA isolated after MELT is suitable for a range of gene expression assays, including quantitative RT-PCR and microarrays.

Additional information is available online (http://www.ambion. $\mathrm{com} / \mathrm{prod} / \mathrm{melt}$ ).

1. Raetz, E.A. \& Moos, P.J I Impact of microarray technology in clinical oncology. Cancer Invest. 22, 312-320 (2004).

2. Bichsel, V.E., Liotta, L.A. \& Petricoin, E.F. Cancer proteomics: from biomarker discovery to signal pathway profiling. Cancer J. 7, 69-78 (2001).

3. de Bono, J.S., Tolcher, A.W. \& Rowinsky, E.K. The future of cytotoxic therapy: selective cytotoxicity based on biology is the key. Breast Cancer Res. $\mathbf{5}$ 154-159 (2003).

4. Van Gelder, R.N. et al. Amplified RNA synthesized from limited quantities of heterogeneous cDNA. Proc Natl. Acad. Sa. USA 87, 1663-1667 (1990). 